\title{
Behavior of an Exponential System of Difference Equations
}

\author{
A. Q. Khan and M. N. Qureshi \\ Department of Mathematics, University of Azad Jammu and Kashmir, Muzaffarabad 13100, Pakistan
}

Correspondence should be addressed to A. Q. Khan; abdulqadeerkhan1@gmail.com

Received 30 April 2014; Accepted 21 July 2014; Published 15 September 2014

Academic Editor: Zhan Zhou

Copyright (C) 2014 A. Q. Khan and M. N. Qureshi. This is an open access article distributed under the Creative Commons Attribution License, which permits unrestricted use, distribution, and reproduction in any medium, provided the original work is properly cited.

\begin{abstract}
We study the qualitative behavior of the following exponential system of rational difference equations: $x_{n+1}=\left(\alpha e^{-y_{n}}+\beta e^{-y_{n-1}}\right) /$ $\left(\gamma+\alpha x_{n}+\beta x_{n-1}\right), y_{n+1}=\left(\alpha_{1} e^{-x_{n}}+\beta_{1} e^{-x_{n-1}}\right) /\left(\gamma_{1}+\alpha_{1} y_{n}+\beta_{1} y_{n-1}\right), n=0,1, \ldots$, where $\alpha, \beta, \gamma, \alpha_{1}, \beta_{1}$, and $\gamma_{1}$ and initial conditions $x_{0}, x_{-1}, y_{o}$, and $y_{-1}$ are positive real numbers. More precisely, we investigate the boundedness character and persistence, existence and uniqueness of positive equilibrium, local and global behavior, and rate of convergence of positive solutions that converges to unique positive equilibrium point of the system. Some numerical examples are given to verify our theoretical results.
\end{abstract}

\section{Introduction}

Mathematical models of population dynamics have created great interest in the field of difference equations. As pointed out in $[1,2]$, to model biological phenomenon, discrete dynamical systems are more appropriate than continuous time models, being computationally efficient to get numerical results. Difference equations also appear naturally as discrete analogs of differential and delay differential equations and have applications in finance, biological, physical, and social sciences. Nonlinear difference equations and their stability analysis and global and local behaviors are of great interest on their own. For some interesting results in this regard we refer to [3-6] and the references therein. Exponential difference equations made their appearance in population dynamics. Though their analysis is hard, it is very interesting at the same time. Biologists believe that the equilibrium points and their stability analysis are important to understand the population dynamics.

El-Metwally et al. [3] have investigated the boundedness character, asymptotic behavior, periodicity nature of the positive solutions, and stability of equilibrium point of the following population model:

$$
x_{n+1}=\alpha+\beta x_{n-1} e^{-x_{n}}, \quad n=0,1, \ldots,
$$

where the parameters $\alpha, \beta$ are positive numbers and the initial conditions are arbitrary nonnegative real numbers.
Ozturk et al. [7] have investigated the boundedness, asymptotic behavior, periodicity, and stability of the positive solutions of the following difference equation:

$$
y_{n+1}=\frac{\alpha+\beta e^{-y_{n}}}{\gamma+y_{n-1}}, \quad n=0,1, \ldots,
$$

where the parameters $\alpha, \beta$, and $\gamma$ are positive numbers and the initial conditions are arbitrary nonnegative numbers.

Bozkurt [8] has investigated the local and global behavior of positive solutions of the following difference equation:

$$
y_{n+1}=\frac{\alpha e^{-y_{n}}+\beta e^{-y_{n-1}}}{\gamma+\alpha y_{n}+\beta y_{n-1}}, \quad n=0,1, \ldots,
$$

where the parameters $\alpha, \beta$, and $\gamma$ and the initial conditions are arbitrary positive numbers.

Motivated by the above studies, our aim in this paper is to investigate the qualitative behavior of positive solutions of the following exponential system of rational difference equations:

$$
\begin{array}{r}
x_{n+1}=\frac{\alpha e^{-y_{n}}+\beta e^{-y_{n-1}}}{\gamma+\alpha x_{n}+\beta x_{n-1}}, \quad y_{n+1}=\frac{\alpha_{1} e^{-x_{n}}+\beta_{1} e^{-x_{n-1}}}{\gamma_{1}+\alpha_{1} y_{n}+\beta_{1} y_{n-1}}, \\
n=0,1, \ldots,
\end{array}
$$

where the parameters $\alpha, \beta, \gamma, \alpha_{1}, \beta_{1}$, and $\gamma_{1}$ are positive numbers and the initial conditions are arbitrary nonnegative real numbers. 
More precisely, we investigate the boundedness character, persistence, existence, and uniqueness of positive steady state, local asymptotic stability and global behavior of unique positive equilibrium point, and rate of convergence of positive solutions of system (4) which converge to its unique positive equilibrium point.

\section{Boundedness and Persistence}

The following theorem shows that every solution of (4) is bounded and persists.

Theorem 1. Every positive solution $\left\{\left(x_{n}, y_{n}\right)\right\}$ of the system (4) is bounded and persists.

Proof. Let $\left\{\left(x_{n}, y_{n}\right)\right\}$ be an arbitrary solution of (4); then

$$
x_{n} \leq \frac{\alpha+\beta}{\gamma}=U_{1}, \quad y_{n} \leq \frac{\alpha_{1}+\beta_{1}}{\gamma_{1}}=U_{2}, \quad n=0,1,2, \ldots
$$

From (4) and (5), we have

$$
\begin{array}{r}
x_{n} \geq \frac{(\alpha+\beta) e^{-\left(\left(\alpha_{1}+\beta_{1}\right) / \gamma_{1}\right)}}{\gamma+(\alpha+\beta)((\alpha+\beta) / \gamma)}=L_{1}, \\
y_{n} \geq \frac{\left(\alpha_{1}+\beta_{1}\right) e^{-((\alpha+\beta) / \gamma)}}{\gamma_{1}+\left(\alpha_{1}+\beta_{1}\right)\left(\left(\alpha_{1}+\beta_{1}\right) / \gamma_{1}\right)}=L_{2}, \\
n=2,3, \ldots .
\end{array}
$$

So, from (5) and (6), we get

$$
L_{1} \leq x_{n} \leq U_{1}, \quad L_{2} \leq y_{n} \leq U_{2}, \quad n=3,4, \ldots .
$$

This proves the statement.

Theorem 2. Let $\left\{\left(x_{n}, y_{n}\right)\right\}$ be a positive solution of the system (4). Then, $\left[L_{1}, U_{1}\right] \times\left[L_{2}, U_{2}\right]$ is invariant set for system (4).

Proof. It follows by induction.

\section{Linearized Stability}

Let us consider four-dimensional discrete dynamical system of the form

$$
\begin{array}{r}
x_{n+1}=f\left(x_{n}, x_{n-1}, y_{n}, y_{n-1}\right), \\
y_{n+1}=g\left(x_{n}, x_{n-1}, y_{n}, y_{n-1}\right), \\
n=0,1, \ldots,
\end{array}
$$

where $f: I^{2} \times J^{2} \rightarrow I$ and $g: I^{2} \times J^{2} \rightarrow J$ are continuously differentiable functions and $I, J$ are some intervals of real numbers. Furthermore, a solution $\left\{\left(x_{n}, y_{n}\right)\right\}_{n=-1}^{\infty}$ of system (8) is uniquely determined by initial conditions $\left(x_{i}, y_{i}\right) \in$ $I \times J$ for $i \in\{-1,0\}$. Along with system (8) we consider the corresponding vector map $F=\left(f, x_{n}, g, y_{n}\right)$. An equilibrium point of $(8)$ is a point $(\bar{x}, \bar{y})$ that satisfies

$$
\begin{aligned}
& \bar{x}=f(\bar{x}, \bar{x}, \bar{y}, \bar{y}), \\
& \bar{y}=g(\bar{x}, \bar{x}, \bar{y}, \bar{y}) .
\end{aligned}
$$

The point $(\bar{x}, \bar{y})$ is also called a fixed point of the vector map $F$.

Definition 3. Let $(\bar{x}, \bar{y})$ be an equilibrium point of the system (8).

(i) An equilibrium point $(\bar{x}, \bar{y})$ is said to be stable if for every $\varepsilon>0$ there exists $\delta>0$ such that for every initial condition $\left(x_{i}, y_{i}\right), i \in\{-1,0\}\left\|\sum_{i=-1}^{0}\left(x_{i}, y_{i}\right)-(\bar{x}, \bar{y})\right\|<$ $\delta$ implies $\left\|\left(x_{n}, y_{n}\right)-(\bar{x}, \bar{y})\right\|<\varepsilon$ for all $n>0$, where $\|\cdot\|$ is the usual Euclidian norm in $\mathbb{R}^{2}$.

(ii) An equilibrium point $(\bar{x}, \bar{y})$ is said to be unstable if it is not stable.

(iii) An equilibrium point $(\bar{x}, \bar{y})$ is said to be asymptotically stable if there exists $\eta>0$ such that $\left\|\sum_{i=-1}^{0}\left(x_{i}, y_{i}\right)-(\bar{x}, \bar{y})\right\|<\eta$ and $\left(x_{n}, y_{n}\right) \rightarrow(\bar{x}, \bar{y})$ as $n \rightarrow \infty$.

(iv) An equilibrium point $(\bar{x}, \bar{y})$ is called global attractor if $\left(x_{n}, y_{n}\right) \rightarrow(\bar{x}, \bar{y})$ as $n \rightarrow \infty$.

(v) An equilibrium point $(\bar{x}, \bar{y})$ is called asymptotic global attractor if it is a global attractor and stable.

Definition 4. Let $(\bar{x}, \bar{y})$ be an equilibrium point of the map

$$
F=\left(f, x_{n}, g, y_{n}\right)
$$

where $f$ and $g$ are continuously differentiable functions at $(\bar{x}, \bar{y})$. The linearized system of (8) about the equilibrium point $(\bar{x}, \bar{y})$ is

$$
X_{n+1}=F\left(X_{n}\right)=F_{J} X_{n},
$$

where $X_{n}=\left(\begin{array}{c}x_{n} \\ x_{n-1} \\ y_{n} \\ y_{n-1}\end{array}\right)$ and $F_{J}$ is the Jacobian matrix of the system (8) about the equilibrium point $(\bar{x}, \bar{y})$.

Let $(\bar{x}, \bar{y})$ be equilibrium point of the system $(4)$; then

$$
\bar{x}=\frac{(\alpha+\beta) e^{-\bar{y}}}{\gamma+(\alpha+\beta) \bar{x}}, \quad \bar{y}=\frac{\left(\alpha_{1}+\beta_{1}\right) e^{-\bar{x}}}{\gamma_{1}+\left(\alpha_{1}+\beta_{1}\right) \bar{y}} .
$$

To construct corresponding linearized form of system (4) we consider the following transformation:

$$
\left(x_{n}, x_{n-1}, y_{n}, y_{n-1}\right) \longmapsto\left(f, f_{1}, g, g_{1}\right),
$$

where

$$
\begin{array}{cc}
f=\frac{\alpha e^{-y_{n}}+\beta e^{-y_{n-1}}}{\gamma+\alpha x_{n}+\beta x_{n-1}}, & f_{1}=x_{n}, \\
g=\frac{\alpha_{1} e^{-x_{n}}+\beta_{1} e^{-x_{n-1}}}{\gamma_{1}+\alpha_{1} y_{n}+\beta_{1} y_{n-1}}, & g_{1}=y_{n} .
\end{array}
$$

The Jacobian matrix about the fixed point $(\bar{x}, \bar{y})$ under the transformation (13) is given by

$$
F_{J}(\bar{x}, \bar{y})=\left(\begin{array}{cccc}
A & B & C & D \\
1 & 0 & 0 & 0 \\
A_{1} & B_{1} & C_{1} & D_{1} \\
0 & 0 & 1 & 0
\end{array}\right)
$$


where

$$
\begin{array}{cc}
A=-\frac{\alpha \bar{x}}{\gamma+(\alpha+\beta) \bar{x}}, & B=-\frac{\beta \bar{x}}{\gamma+(\alpha+\beta) \bar{x}}, \\
C=-\frac{\alpha e^{-\bar{y}}}{\gamma+(\alpha+\beta) \bar{x}}, & D=-\frac{\beta e^{-\bar{y}}}{\gamma+(\alpha+\beta) \bar{x}}, \\
A_{1}=-\frac{\alpha_{1} e^{-\bar{x}}}{\gamma_{1}+\left(\alpha_{1}+\beta_{1}\right) \bar{y}}, & B_{1}=-\frac{\beta_{1} e^{-\bar{x}}}{\gamma_{1}+\left(\alpha_{1}+\beta_{1}\right) \bar{y}}, \\
C_{1}=-\frac{\alpha_{1} \bar{y}}{\gamma_{1}+\left(\alpha_{1}+\beta_{1}\right) \bar{y}}, & D_{1}=-\frac{\beta_{1} \bar{y}}{\gamma_{1}+\left(\alpha_{1}+\beta_{1}\right) \bar{y}} .
\end{array}
$$

Lemma 5 (see [9]). For the system $X_{n+1}=F\left(X_{n}\right), n=0,1, \ldots$, of difference equations such that $\bar{X}$ is a fixed point of $F$. If all eigenvalues of the Jacobian matrix $J_{F}$ about $\bar{X}$ lie inside the open unit disk $|\lambda|<1$, then $\bar{X}$ is locally asymptotically stable. If one of them has a modulus greater than one, then $\bar{X}$ is unstable.

The following theorem shows the existence and uniqueness of positive equilibrium point of system (4).

Theorem 6. If

$$
\begin{aligned}
\zeta< & \left(\gamma_{1}+\left(\alpha_{1}+\beta_{1}\right) \ln \left[\frac{\alpha+\beta}{\left(\gamma+(\alpha+\beta) L_{1}\right) L_{1}}\right]\right) \\
& \times \ln \left[\frac{\alpha+\beta}{\left(\gamma+(\alpha+\beta) L_{1}\right) L_{1}}\right],
\end{aligned}
$$

where $\zeta=\gamma_{1}\left(\left(\left(\gamma+2(\alpha+\beta) U_{1}\right) /\left(\left(\gamma+(\alpha+\beta) L_{1}\right) L_{1}\right)\right)-\ln [(\alpha+\right.$ $\left.\left.\beta) /\left(\left(\gamma+(\alpha+\beta) L_{1}\right) L_{1}\right)\right]\right)+2\left(\alpha_{1}+\beta_{1}\right)\left(\left(\gamma+2(\alpha+\beta) U_{1}\right) /((\gamma+\right.$ $\left.\left.\left.(\alpha+\beta) L_{1}\right) L_{1}\right)\right) \ln \left[(\alpha+\beta) /\left(\left(\gamma+(\alpha+\beta) L_{1}\right) L_{1}\right)\right]$, then the system (4) has a unique positive equilibrium point $(\bar{x}, \bar{y})$ in $\left[L_{1}, U_{1}\right] \times$ $\left[L_{2}, U_{2}\right]$.

Proof. Consider the following system of algebraic equations:

$$
x=\frac{(\alpha+\beta) e^{-y}}{\gamma+(\alpha+\beta) x}, \quad y=\frac{\left(\alpha_{1}+\beta_{1}\right) e^{-x}}{\gamma_{1}+\left(\alpha_{1}+\beta_{1}\right) y} .
$$

Assume that $(x, y) \in\left[L_{1}, U_{1}\right] \times\left[L_{2}, U_{2}\right]$; then it follows from (18) that

$$
\begin{gathered}
y=\ln \left[\frac{\alpha+\beta}{(\gamma+(\alpha+\beta) x) x}\right], \\
x=\ln \left[\frac{\alpha_{1}+\beta_{1}}{\left(\gamma_{1}+\left(\alpha_{1}+\beta_{1}\right) y\right) y}\right] .
\end{gathered}
$$

Define

$$
F(x)=\ln \left[\frac{\alpha_{1}+\beta_{1}}{\left(\gamma_{1}+\left(\alpha_{1}+\beta_{1}\right) h(x)\right) h(x)}\right]-x,
$$

where $h(x)=\ln [(\alpha+\beta) /((\gamma+(\alpha+\beta) x) x)], x \in\left[L_{1}, U_{1}\right]$. It is easy to see that

$$
F\left(L_{1}\right)=\ln \left[\frac{\alpha_{1}+\beta_{1}}{\left(\gamma_{1}+\left(\alpha_{1}+\beta_{1}\right) h\left(L_{1}\right)\right) h\left(L_{1}\right)}\right]-L_{1}>0
$$

if and only if

$$
\begin{aligned}
& \ln \left[( \alpha _ { 1 } + \beta _ { 1 } ) \left(\left(\gamma_{1}+\left(\alpha_{1}+\beta_{1}\right) \ln \left[\frac{\alpha+\beta}{\left(\gamma+(\alpha+\beta) L_{1}\right) L_{1}}\right]\right)\right.\right. \\
& \left.\left.\quad \times \ln \left[\frac{\alpha+\beta}{\left(\gamma+(\alpha+\beta) L_{1}\right) L_{1}}\right]\right)^{-1}\right] \\
& >L_{1}, \\
& F\left(U_{1}\right)=\ln \left[\frac{\alpha_{1}+\beta_{1}}{\left(\gamma_{1}+\left(\alpha_{1}+\beta_{1}\right) h\left(U_{1}\right) h\left(U_{1}\right)\right.}\right]-U_{1}<0
\end{aligned}
$$

if and only if

$$
\begin{gathered}
\ln \left[( \alpha _ { 1 } + \beta _ { 1 } ) \left(\left(\gamma_{1}+\left(\alpha_{1}+\beta_{1}\right) \ln \left[\frac{\alpha+\beta}{\left(\gamma+(\alpha+\beta) U_{1}\right) U_{1}}\right]\right)\right.\right. \\
\left.\left.\times \ln \left[\frac{\alpha+\beta}{\left(\gamma+(\alpha+\beta) U_{1}\right) U_{1}}\right]\right)^{-1}\right]
\end{gathered}
$$

$<U_{1}$.

Hence, $F(x)$ has at least one positive solution in the interval $\left[L_{1}, U_{1}\right]$. Furthermore, assume that condition (17) is satisfied; then one has

$$
\begin{aligned}
& F^{\prime}(x)=\left(\left(\gamma _ { 1 } \left(\frac{\gamma+2(\alpha+\beta) x}{(\gamma+(\alpha+\beta) x) x}\right.\right.\right. \\
& \left.-\ln \left[\frac{\alpha+\beta}{(\gamma+(\alpha+\beta) x) x}\right]\right) \\
& +2\left(\alpha_{1}+\beta_{1}\right) \frac{\gamma+2(\alpha+\beta) x}{(\gamma+(\alpha+\beta) x) x} \\
& \left.\times \ln \left[\frac{\alpha+\beta}{(\gamma+(\alpha+\beta) x) x}\right]\right) \\
& \times\left(\left(\gamma_{1}+\left(\alpha_{1}+\beta_{1}\right) \ln \left[\frac{\alpha+\beta}{(\gamma+(\alpha+\beta) x) x}\right]\right)\right. \\
& \left.\left.\times \ln \left[\frac{\alpha+\beta}{(\gamma+(\alpha+\beta) x) x}\right]\right)^{-1}\right)-1 \\
& \leq\left(\gamma _ { 1 } \left(\frac{\gamma+2(\alpha+\beta) U_{1}}{\left(\gamma+(\alpha+\beta) L_{1}\right) L_{1}}\right.\right. \\
& \left.-\ln \left[\frac{\alpha+\beta}{\left(\gamma+(\alpha+\beta) L_{1}\right) L_{1}}\right]\right) \\
& +2\left(\alpha_{1}+\beta_{1}\right) \frac{\gamma+2(\alpha+\beta) U_{1}}{\left(\gamma+(\alpha+\beta) L_{1}\right) L_{1}} \\
& \left.\times \ln \left[\frac{\alpha+\beta}{\left(\gamma+(\alpha+\beta) L_{1}\right) L_{1}}\right]\right)
\end{aligned}
$$




$$
\begin{gathered}
\times\left(\left(\gamma_{1}+\left(\alpha_{1}+\beta_{1}\right) \ln \left[\frac{\alpha+\beta}{\left(\gamma+(\alpha+\beta) L_{1}\right) L_{1}}\right]\right)\right. \\
\left.\left.\times \ln \left[\frac{\alpha+\beta}{\left(\gamma+(\alpha+\beta) L_{1}\right) L_{1}}\right]\right)^{-1}\right)-1<0 .
\end{gathered}
$$

Hence, $F(x)=0$ has a unique positive solution in $\left[L_{1}, U_{1}\right]$. This completes the proof.

Theorem 7. If

$$
\mu<\left(\gamma+(\alpha+\beta) L_{1}\right)\left(\gamma_{1}+\left(\alpha_{1}+\beta_{1}\right) L_{2}\right)
$$

where

$$
\begin{aligned}
\mu= & (\alpha+\beta) U_{1}\left(\gamma_{1}+\left(\alpha_{1}+\beta_{1}\right) L_{2}\right) \\
& +\left(\alpha_{1}+\beta_{1}\right) U_{2}\left(\gamma+(\alpha+\beta) L_{1}\right) \\
& +(\alpha+\beta)\left(\alpha_{1}+\beta_{1}\right)\left(e^{-L_{1}-L_{2}}+U_{1} U_{2}\right),
\end{aligned}
$$

then, the unique positive equilibrium point $(\bar{x}, \bar{y})$ of the system (4) is locally asymptotically stable.

Proof. The characteristic equation of the Jacobian matrix $F_{J}(\bar{x}, \bar{y})$ about equilibrium point $(\bar{x}, \bar{y})$ is given by

$$
\lambda^{4}-p_{4} \lambda^{3}+p_{3} \lambda^{2}+p_{2} \lambda+p_{1}=0,
$$

where $p_{4}=A+C_{1}, p_{3}=A C_{1}-B-A_{1} C-D_{1}, p_{2}=A D_{1}-$ $A_{1} D+B C_{1}-B_{1} C$, and $p_{1}=B D_{1}-B_{1} D$.

Assuming condition (25) one has

$$
\begin{aligned}
& \sum_{i=1}^{4}\left|p_{i}\right| \\
& =\frac{(\alpha+\beta) \bar{x}}{\gamma+(\alpha+\beta) \bar{x}}+\frac{\left(\alpha_{1}+\beta_{1}\right) \bar{y}}{\gamma_{1}+\left(\alpha_{1}+\beta_{1}\right) \bar{y}} \\
& +\left(\left(\left(\alpha \alpha_{1}+\alpha \beta_{1}+\alpha_{1} \beta+\beta \beta_{1}\right) e^{-\bar{x}-\bar{y}}\right.\right. \\
& \left.+\left(\alpha \alpha_{1}+\alpha \beta_{1}+\alpha_{1} \beta+\beta \beta_{1}\right) \overline{x y}\right) \\
& \left.\times\left((\gamma+(\alpha+\beta) \bar{x})\left(\gamma_{1}+\left(\alpha_{1}+\beta_{1}\right) \bar{y}\right)\right)^{-1}\right) \\
& =\frac{(\alpha+\beta) \bar{x}}{\gamma+(\alpha+\beta) \bar{x}}+\frac{\left(\alpha_{1}+\beta_{1}\right) \bar{y}}{\gamma_{1}+\left(\alpha_{1}+\beta_{1}\right) \bar{y}} \\
& +\frac{(\alpha+\beta)\left(\alpha_{1}+\beta_{1}\right)\left(e^{-\bar{x}-\bar{y}}+\overline{x y}\right)}{(\gamma+(\alpha+\beta) \bar{x})\left(\gamma_{1}+\left(\alpha_{1}+\beta_{1}\right) \bar{y}\right)} \\
& <\frac{(\alpha+\beta) U_{1}}{\gamma+(\alpha+\beta) L_{1}}+\frac{\left(\alpha_{1}+\beta_{1}\right) U_{2}}{\gamma_{1}+\left(\alpha_{1}+\beta_{1}\right) L_{2}} \\
& +\frac{(\alpha+\beta)\left(\alpha_{1}+\beta_{1}\right)\left(e^{-L_{1}-L_{2}}+U_{1} U_{2}\right)}{\left(\gamma+(\alpha+\beta) L_{1}\right)\left(\gamma_{1}+\left(\alpha_{1}+\beta_{1}\right) L_{2}\right)} \\
& =\frac{\mu}{\left(\gamma+(\alpha+\beta) L_{1}\right)\left(\gamma_{1}+\left(\alpha_{1}+\beta_{1}\right) L_{2}\right)}
\end{aligned}
$$

$<1$.
Therefore, inequality (28) and Remark 1.3.1 of reference [10] imply that the unique positive equilibrium point $(\bar{x}, \bar{y})$ of the system (4) is locally asymptotically stable.

\section{Global Character}

Lemma 8 (see [11]). Let $I=[a, b]$ and $J=[c, d]$ be real intervals, and let $f: I \times J \rightarrow I$ and $g: I \times J \rightarrow J$ be continuous functions. Consider the system (8) with initial conditions $x_{0}, x_{-1}, y_{o}, y_{-1} \in I \times J$. Suppose that the following statements are true.

(i) $f(x, y)$ is nonincreasing in both arguments $x, y$.

(ii) $g(x, y)$ is nonincreasing in both arguments $x, y$.

(iii) If $\left(m_{1}, M_{1}, m_{2}, M_{2}\right) \in I \times J$ is a solution of the system

$$
\begin{array}{ll}
m_{1}=f\left(M_{1}, M_{2}\right), & M_{1}=f\left(m_{1}, m_{2}\right) \\
m_{2}=g\left(M_{1}, M_{2}\right), & M_{2}=g\left(m_{1}, m_{2}\right)
\end{array}
$$

such that $m_{1}=M_{1}$ and $m_{2}=M_{2}$, then there exists exactly one equilibrium point $(\bar{x}, \bar{y})$ of the system (8) such that $\lim _{n \rightarrow \infty}\left(x_{n}, y_{n}\right)=(\bar{x}, \bar{y})$.

Theorem 9. The unique positive equilibrium point $(\bar{x}, \bar{y})$ of the system (4) is a global attractor.

Proof. Define $f(x, y)=\left((\alpha+\beta) e^{-y}\right) /(\gamma+(\alpha+\beta) x)$ and $g(x, y)=\left(\left(\alpha_{1}+\beta_{1}\right) e^{-x}\right) /\left(\gamma_{1}+\left(\alpha_{1}+\beta_{1}\right) y\right)$. It is easy to see that $f(x, y)$ and $g(x, y)$ are nonincreasing in both arguments $x$ and $y$. Let $\left(m_{1}, M_{1}, m_{2}, M_{2}\right) \in I \times J$ be a solution of the system

$$
\begin{array}{ll}
m_{1}=f\left(M_{1}, M_{2}\right), & M_{1}=f\left(m_{1}, m_{2}\right) \\
m_{2}=g\left(M_{1}, M_{2}\right), & M_{2}=g\left(m_{1}, m_{2}\right) .
\end{array}
$$

Then, one has

$$
\begin{array}{ll}
m_{1}=\frac{(\alpha+\beta) e^{-M_{2}}}{\gamma+(\alpha+\beta) M_{1}}, & M_{1}=\frac{(\alpha+\beta) e^{-m_{2}}}{\gamma+(\alpha+\beta) m_{1}}, \\
m_{2}=\frac{\left(\alpha_{1}+\beta_{1}\right) e^{-M_{1}}}{\gamma_{1}+\left(\alpha_{1}+\beta_{1}\right) M_{2}}, & M_{2}=\frac{\left(\alpha_{1}+\beta_{1}\right) e^{-m_{1}}}{\gamma_{1}+\left(\alpha_{1}+\beta_{1}\right) m_{2}} .
\end{array}
$$

Furthermore, arguing as in the proof of Theorem 1.16 of [11], it suffices to suppose that

$$
m_{1} \leq M_{1}, \quad m_{2} \leq M_{2}
$$

Moreover,

$$
\begin{array}{ll}
L_{1} \leq m_{1}, & M_{1} \leq U_{1}, \\
L_{2} \leq m_{2}, & M_{2} \leq U_{2} .
\end{array}
$$


From (31), we have

$$
\begin{aligned}
m_{1}- & M_{1} \\
= & \left(\gamma(\alpha+\beta)\left(e^{-M_{2}}-e^{-m_{2}}\right)\right. \\
& \left.+(\alpha+\beta)^{2}\left(m_{1} e^{-M_{2}}-M_{1} e^{-m_{2}}\right)\right) \\
& \times\left(\left(\gamma+(\alpha+\beta) M_{1}\right)\left(\gamma+(\alpha+\beta) m_{1}\right)\right)^{-1} .
\end{aligned}
$$

Using the fact that $e^{-M_{2}} \leq e^{-m_{2}}$, one gets

$$
m_{1}-M_{1} \leq \frac{(\alpha+\beta)^{2} e^{-L_{2}}}{\left(\gamma+(\alpha+\beta) L_{1}\right)^{2}}\left(m_{1}-M_{1}\right) .
$$

From (36), we conclude that $m_{1}=M_{1}$. Similarly, from (32), it is easy to show that $m_{2}=M_{2}$. Hence, from Lemma 8 , the unique positive equilibrium point $(\bar{x}, \bar{y})$ of the system (4) is a global attractor.

Corollary 10. If condition (25) of Theorem 7 is satisfied, then the unique positive equilibrium point $(\bar{x}, \bar{y})$ of the system (4) is globally asymptotically stable.

Proof. The proof is a direct consequence of Theorems 7 and 9.

\section{Rate of Convergence}

In this section we will determine the rate of convergence of a solution that converges to the unique positive equilibrium point of the system (4).

The following result gives the rate of convergence of solutions of a system of difference equations:

$$
X_{n+1}=(A+B(n)) X_{n},
$$

where $X_{n}$ is an $m$-dimensional vector, $A \in C^{m \times m}$ is a constant matrix, and $B: \mathbb{Z}^{+} \rightarrow C^{m \times m}$ is a matrix function satisfying

$$
\|B(n)\| \longrightarrow 0
$$

as $n \rightarrow \infty$, where $\|\cdot\|$ denotes any matrix norm which is associated with the vector norm

$$
\|(x, y)\|=\sqrt{x^{2}+y^{2}} .
$$

Proposition 11 (Perron's theorem [12]). Suppose that condition (38) holds. If $X_{n}$ is a solution of (37), then either $X_{n}=0$ for all large $n$ or

$$
\rho=\lim _{n \rightarrow \infty}\left(\left\|X_{n}\right\|\right)^{1 / n}
$$

exists and is equal to the modulus of one of the eigenvalues of matrix $A$.

Proposition 12 (see [12]). Suppose that condition (38) holds. If $X_{n}$ is a solution of (37), then either $X_{n}=0$ for all large $n$ or

$$
\rho=\lim _{n \rightarrow \infty} \frac{\left\|X_{n+1}\right\|}{\left\|X_{n}\right\|}
$$

exists and is equal to the modulus of one of the eigenvalues of matrix $A$.
Let $\left\{\left(x_{n}, y_{n}\right)\right\}$ be any solution of the system (4) such that $\lim _{n \rightarrow \infty} x_{n}=\bar{x}$ and $\lim _{n \rightarrow \infty} y_{n}=\bar{y}$. To find the error terms, one has from the system (4)

$$
\begin{aligned}
x_{n+1}-\bar{x}= & \frac{\alpha e^{-y_{n}}+\beta e^{-y_{n-1}}}{\gamma+\alpha x_{n}+\beta x_{n-1}}-\frac{(\alpha+\beta) e^{-\bar{y}}}{\gamma+(\alpha+\beta) \bar{x}}, \\
= & -\frac{\alpha \bar{x}\left(x_{n}-\bar{x}\right)}{\gamma+\alpha x_{n}+\beta x_{n-1}}-\frac{\beta \bar{x}\left(x_{n-1}-\bar{x}\right)}{\gamma+\alpha x_{n}+\beta x_{n-1}} \\
& -\frac{\alpha e^{-y_{n}}\left(e^{y_{n}-\bar{y}}-1\right)}{\gamma+\alpha x_{n}+\beta x_{n-1}}-\frac{\beta e^{-y_{n-1}}\left(e^{y_{n-1}-\bar{y}}-1\right)}{\gamma+\alpha x_{n}+\beta x_{n-1}}, \\
= & -\frac{\alpha \bar{x}\left(x_{n}-\bar{x}\right)}{\gamma+\alpha x_{n}+\beta x_{n-1}}-\frac{\beta \bar{x}\left(x_{n-1}-\bar{x}\right)}{\gamma+\alpha x_{n}+\beta x_{n-1}} \\
& -\frac{\alpha e^{-y_{n}}\left(y_{n}-\bar{y}+O_{1}\left(y_{n}-\bar{y}\right)^{2}\right)}{\gamma+\alpha x_{n}+\beta x_{n-1}} \\
& -\frac{\beta e^{-y_{n-1}\left(y_{n-1}-\bar{y}+O_{2}\left(\left(y_{n-1}-\bar{y}\right)^{2}\right)\right)}}{\gamma+\alpha x_{n}+\beta x_{n-1}} .
\end{aligned}
$$

So,

$$
\begin{aligned}
x_{n+1}-\bar{x}= & -\frac{\alpha \bar{x}}{\gamma+\alpha x_{n}+\beta x_{n-1}}\left(x_{n}-\bar{x}\right) \\
& -\frac{\beta \bar{x}}{\gamma+\alpha x_{n}+\beta x_{n-1}}\left(x_{n-1}-\bar{x}\right) \\
& -\frac{\alpha e^{-y_{n}}}{\gamma+\alpha x_{n}+\beta x_{n-1}}\left(y_{n}-\bar{y}\right) \\
& -\frac{\beta e^{-y_{n-1}}}{\gamma+\alpha x_{n}+\beta x_{n-1}}\left(y_{n-1}-\bar{y}\right) \\
& +O_{1}\left(\left(y_{n}-\bar{y}\right)^{2}\right)+O_{2}\left(\left(y_{n-1}-\bar{y}\right)^{2}\right) .
\end{aligned}
$$

Similarly,

$$
\begin{aligned}
y_{n+1}-\bar{y}= & -\frac{\alpha_{1} e^{-x_{n}}}{\gamma_{1}+\alpha_{1} y_{n}+\beta_{1} y_{n-1}}\left(x_{n}-\bar{x}\right) \\
& -\frac{\beta_{1} e^{-x_{n-1}}}{\gamma_{1}+\alpha_{1} y_{n}+\beta_{1} y_{n-1}}\left(x_{n-1}-\bar{x}\right) \\
& -\frac{\alpha_{1} \bar{y}}{\gamma_{1}+\alpha_{1} y_{n}+\beta_{1} y_{n-1}}\left(y_{n}-\bar{y}\right) \\
& -\frac{\beta_{1} \bar{y}}{\gamma_{1}+\alpha_{1} y_{n}+\beta_{1} x_{n-1}}\left(y_{n-1}-\bar{x}\right) \\
& +O_{3}\left(\left(x_{n}-\bar{y}\right)^{2}\right)+O_{4}\left(\left(x_{n-1}-\bar{y}\right)^{2}\right) .
\end{aligned}
$$


From (43) and (44), we have

$$
\begin{aligned}
x_{n+1}-\bar{x} \approx & -\frac{\alpha \bar{x}}{\gamma+\alpha x_{n}+\beta x_{n-1}}\left(x_{n}-\bar{x}\right) \\
& -\frac{\beta \bar{x}}{\gamma+\alpha x_{n}+\beta x_{n-1}}\left(x_{n-1}-\bar{x}\right) \\
& -\frac{\alpha e^{-y_{n}}}{\gamma+\alpha x_{n}+\beta x_{n-1}}\left(y_{n}-\bar{y}\right) \\
& -\frac{\beta e^{-y_{n-1}}}{\gamma+\alpha x_{n}+\beta x_{n-1}}\left(y_{n-1}-\bar{y}\right), \\
y_{n+1}-\bar{y} \approx & -\frac{\alpha_{1} e^{-x_{n}}}{\gamma_{1}+\alpha_{1} y_{n}+\beta_{1} y_{n-1}}\left(x_{n}-\bar{x}\right) \\
& -\frac{\beta_{1} e^{-x_{n-1}}}{\gamma_{1}+\alpha_{1} y_{n}+\beta_{1} y_{n-1}}\left(x_{n-1}-\bar{x}\right) \\
& -\frac{\alpha_{1} \bar{y}}{\gamma_{1}+\alpha_{1} y_{n}+\beta_{1} y_{n-1}}\left(y_{n}-\bar{y}\right) \\
& -\frac{\beta_{1} \bar{y}}{\gamma_{1}+\alpha_{1} y_{n}+\beta_{1} x_{n-1}}\left(y_{n-1}-\bar{x}\right) .
\end{aligned}
$$

Let $e_{n}^{1}=x_{n}-\bar{x}$ and $e_{n}^{2}=y_{n}-\bar{y}$. Then system (45) can be represented as

$$
\begin{aligned}
& e_{n+1}^{1} \approx a_{n} e_{n}^{1}+b_{n} e_{n-1}^{1}+c_{n} e_{n}^{2}+d_{n} e_{n-1}^{2}, \\
& e_{n+1}^{2} \approx e_{n} e_{n}^{1}+f_{n} e_{n-1}^{1}+g_{n} e_{n}^{2}+h_{n} e_{n-1}^{2},
\end{aligned}
$$

$$
\left(\begin{array}{c}
e_{n+1}^{1} \\
e_{n}^{1} \\
e_{n+1}^{2} \\
e_{n}^{2}
\end{array}\right)=\left(\begin{array}{cc}
-\frac{\alpha \bar{x}}{\gamma+(\alpha+\beta) \bar{x}} & -\frac{\beta \bar{x}}{\gamma+(\alpha+\beta) \bar{x}} \\
1 & 0 \\
-\frac{\alpha_{1} e^{-\bar{x}}}{\gamma_{1}+\left(\alpha_{1}+\beta_{1}\right) \bar{y}} & -\frac{\beta_{1} e^{-\bar{x}}}{\gamma_{1}+\left(\alpha_{1}+\beta_{1}\right) \bar{y}} \\
0 & 0
\end{array}\right.
$$

which is similar to linearized system of (4) about the equilibrium point $(\bar{x}, \bar{y})$. Using Proposition 11 , one has the following result.

Theorem 13. Assume that $\left\{\left(x_{n}, y_{n}\right)\right\}$ is a positive solution of the system (4) such that $\lim _{n \rightarrow \infty} x_{n}=\bar{x}$ and $\lim _{n \rightarrow \infty} y_{n}=\bar{y}$, with $\bar{x}$ in $\left[L_{1}, U_{1}\right]$ and $\bar{y}$ in $\left[L_{2}, U_{2}\right]$. Then, the error vector $e_{n}=$ $\left(\begin{array}{c}e_{n}^{1} \\ e_{n-1}^{1} \\ e_{n}^{2} \\ e_{n-2}^{2}\end{array}\right)$ of every solution of (4) satisfies both of the following asymptotic relations:

$$
\begin{gathered}
\lim _{n \rightarrow \infty}\left(\left\|e_{n}\right\|\right)^{1 / n}=\left|\lambda_{1,2,3,4} F_{J}(\bar{x}, \bar{y})\right|, \\
\lim _{n \rightarrow \infty} \frac{\left\|e_{n+1}\right\|}{\left\|e_{n}\right\|}=\left|\lambda_{1,2,3,4} F_{J}(\bar{x}, \bar{y})\right|,
\end{gathered}
$$

where

$$
\begin{aligned}
a_{n}=-\frac{\alpha \bar{x}}{\gamma+\alpha x_{n}+\beta x_{n-1}}, & b_{n}=-\frac{\beta \bar{x}}{\gamma+\alpha x_{n}+\beta x_{n-1}}, \\
c_{n}=-\frac{\alpha e^{-y_{n}}}{\gamma+\alpha x_{n}+\beta x_{n-1}}, & d_{n}=-\frac{\beta e^{-y_{n-1}}}{\gamma+\alpha x_{n}+\beta x_{n-1}}, \\
e_{n}=-\frac{\alpha_{1} e^{-x_{n}}}{\gamma_{1}+\alpha_{1} y_{n}+\beta_{1} y_{n-1}}, & f_{n}=-\frac{\beta_{1} e^{-x_{n-1}}}{\gamma_{1}+\alpha_{1} y_{n}+\beta_{1} y_{n-1}}, \\
g_{n}=-\frac{\alpha_{1} \bar{y}}{\gamma_{1}+\alpha_{1} y_{n}+\beta_{1} y_{n-1}}, & h_{n}=-\frac{\beta_{1} \bar{y}}{\gamma_{1}+\alpha_{1} y_{n}+\beta_{1} x_{n-1}} .
\end{aligned}
$$

Moreover,

$$
\begin{gathered}
\lim _{n \rightarrow \infty} a_{n}=-\frac{\alpha \bar{x}}{\gamma+(\alpha+\beta) \bar{x}}, \quad \lim _{n \rightarrow \infty} b_{n}=-\frac{\beta \bar{x}}{\gamma+(\alpha+\beta) \bar{x}}, \\
\lim _{n \rightarrow \infty} c_{n}=-\frac{\alpha e^{-\bar{y}}}{\gamma+(\alpha+\beta) \bar{x}}, \quad \lim _{n \rightarrow \infty} d_{n}=-\frac{\beta e^{-\bar{y}}}{\gamma+(\alpha+\beta) \bar{x}}, \\
\lim _{n \rightarrow \infty} e_{n}=-\frac{\alpha_{1} e^{-\bar{x}}}{\gamma_{1}+\left(\alpha_{1}+\beta_{1}\right) \bar{y}}, \\
\lim _{n \rightarrow \infty} f_{n}=-\frac{\beta_{1} e^{-\bar{x}}}{\gamma_{1}+\left(\alpha_{1}+\beta_{1}\right) \bar{y}}, \\
\lim _{n \rightarrow \infty} g_{n}=-\frac{\alpha_{1} \bar{y}}{\gamma_{1}+\left(\alpha_{1}+\beta_{1}\right) \bar{y}} \\
\lim _{n \rightarrow \infty} h_{n}=-\frac{\beta_{1} \bar{y}}{\gamma_{1}+\left(\alpha_{1}+\beta_{1}\right) \bar{y}} .
\end{gathered}
$$

So, the limiting system of error terms can be written as

$$
\left.\begin{array}{cc}
-\frac{\alpha e^{-\bar{y}}}{\gamma+(\alpha+\beta) \bar{x}} & -\frac{\beta e^{-\bar{y}}}{\gamma+(\alpha+\beta) \bar{x}} \\
0 & 0 \\
-\frac{\alpha_{1} \bar{y}}{\gamma_{1}+\left(\alpha_{1}+\beta_{1}\right) \bar{y}} & -\frac{\beta_{1} \bar{y}}{\gamma_{1}+\left(\alpha_{1}+\beta_{1}\right) \bar{y}} \\
1 & 0
\end{array}\right)\left(\begin{array}{c}
e_{n}^{1} \\
e_{n-1}^{1} \\
e_{n}^{2} \\
e_{n-2}^{2}
\end{array}\right) \text {, }
$$

where $\lambda_{1,2,3,4} F_{J}(\bar{x}, \bar{y})$ are the characteristic roots of Jacobian matrix $F_{J}(\bar{x}, \bar{y})$

\section{Examples}

In order to verify our theoretical results and to support our theoretical discussions, we consider several interesting numerical examples in this section. These examples represent different types of qualitative behavior of solutions to the system of nonlinear difference equations (4). All plots in this section are drawn with Mathematica.

Example 1. Let $\alpha=90, \beta=25, \gamma=312, \alpha_{1}=2, \beta_{1}=30$, and $\gamma_{1}=5$. Then, the system (4) can be written as

$$
x_{n+1}=\frac{90 e^{-y_{n}}+25 e^{-y_{n-1}}}{312+90 x_{n}+25 x_{n-1}}, \quad y_{n+1}=\frac{2 e^{-x_{n}}+30 e^{-x_{n-1}}}{5+2 y_{n}+30 y_{n-1}},
$$




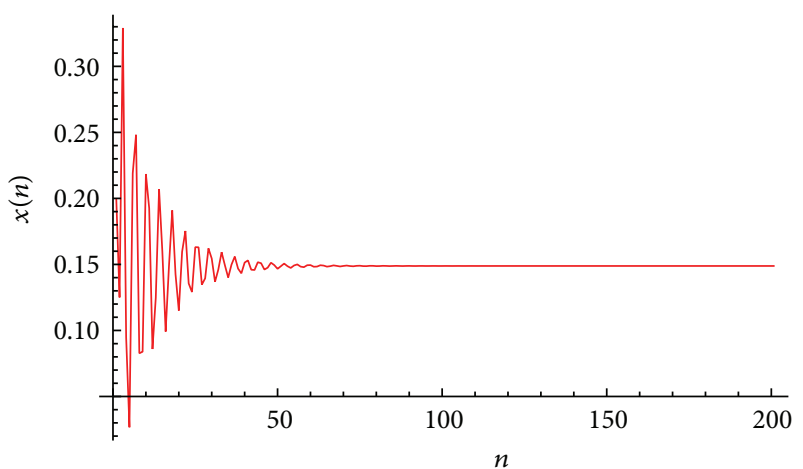

Figure 1: Plot of $x_{n}$ for the system (51).

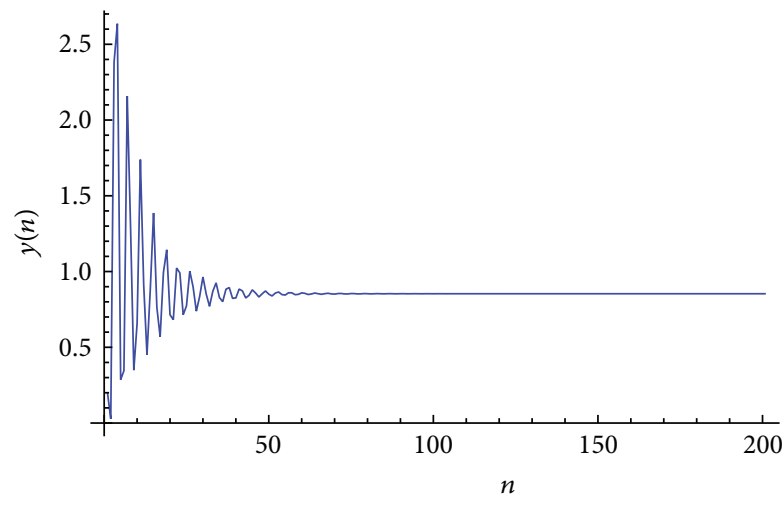

Figure 2: Plot of $y_{n}$ for the system (51).

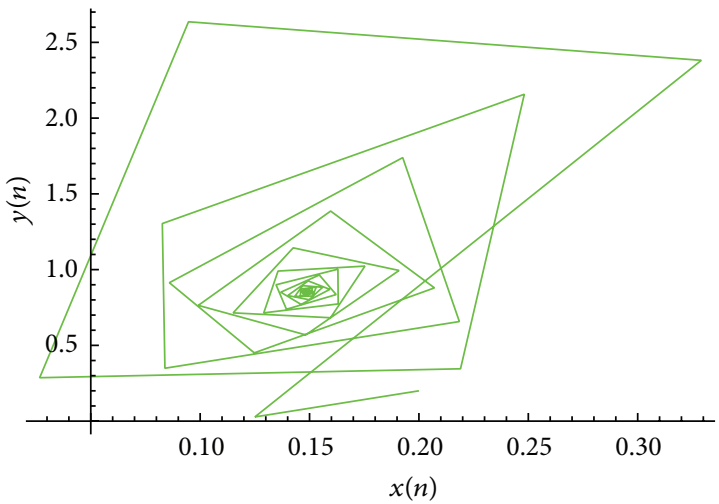

FIgURE 3: An attractor of the system (51).

with initial conditions $x_{-1}=11.7, x_{0}=0.2, y_{-1}=1.8$, and $y_{0}=0.2$.

The plot of $x_{n}$ for the system (51) is shown in Figure 1, plot of $y_{n}$ for the system (51) is shown in Figure 2, and its global attractor is shown in Figure 3. The unique positive equilibrium point of the system (51) is given by $(\bar{x}, \bar{y})=$ $(0.148835,0.853441)$.

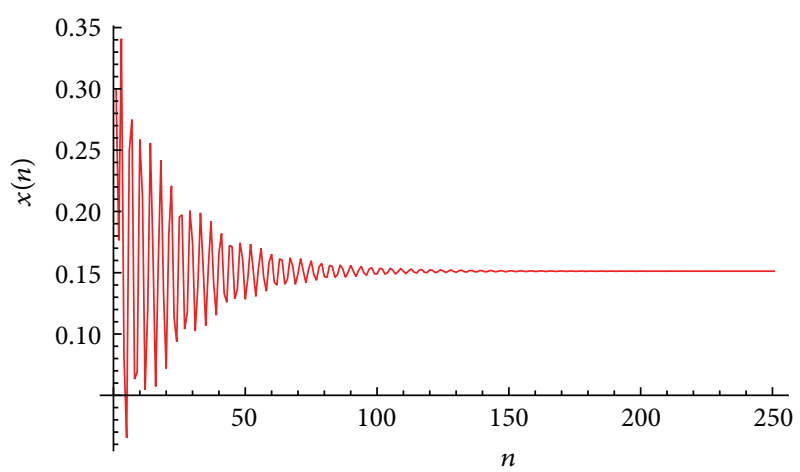

Figure 4: Plot of $x_{n}$ for the system (52).

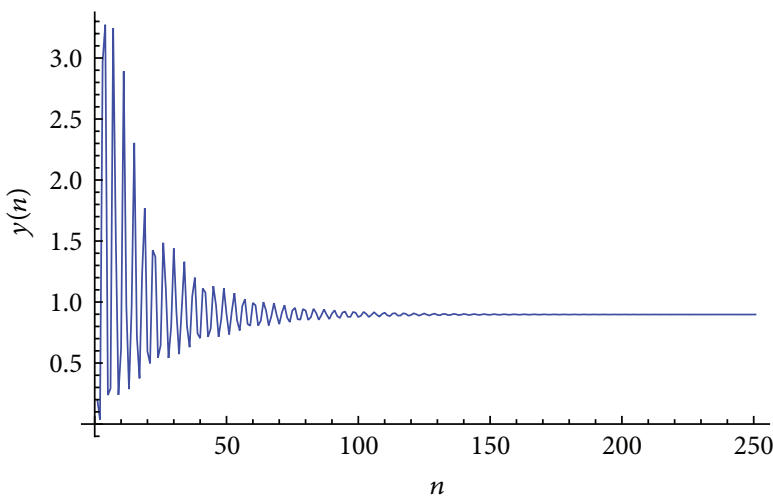

Figure 5: Plot of $y_{n}$ for the system (52).

Example 2. Let $\alpha=225, \beta=55, \gamma=712, \alpha_{1}=1.7, \beta_{1}=30$, and $\gamma_{1}=1.9$. Then, the system (4) can be written as

$$
\begin{aligned}
x_{n+1} & =\frac{225 e^{-y_{n}}+55 e^{-y_{n-1}}}{712+225 x_{n}+55 x_{n-1}}, \\
y_{n+1} & =\frac{1.7 e^{-x_{n}}+30 e^{-x_{n-1}}}{1.9+1.7 y_{n}+30 y_{n-1}},
\end{aligned}
$$

with initial conditions $x_{-1}=6.7, x_{0}=0.3, y_{-1}=1.1$, and $y_{0}=0.2$.

The plot of $x_{n}$ for the system (52) is shown in Figure 4, plot of $y_{n}$ for the system (52) is shown in Figure 5, and its global attractor is shown in Figure 6. The unique positive equilibrium point of the system (52) is given by $(\bar{x}, \bar{y})=$ $(0.151261,0.897674)$.

Example 3. Let $\alpha=125, \beta=14, \gamma=112, \alpha_{1}=15, \beta_{1}=32$, and $\gamma_{1}=0.009$. Then, the system (4) can be written as

$$
\begin{aligned}
& x_{n+1}=\frac{125 e^{-y_{n}}+14 e^{-y_{n-1}}}{112+125 x_{n}+14 x_{n-1}}, \\
& y_{n+1}=\frac{15 e^{-x_{n}}+32 e^{-x_{n-1}}}{0.009+15 y_{n}+32 y_{n-1}},
\end{aligned}
$$

with initial conditions $x_{-1}=0.7, x_{0}=0.3, y_{-1}=0.9$, and $y_{0}=0.2$.

The plot of $x_{n}$ for the system (53) is shown in Figure 7, plot of $y_{n}$ for the system (53) is shown in Figure 8, and its 


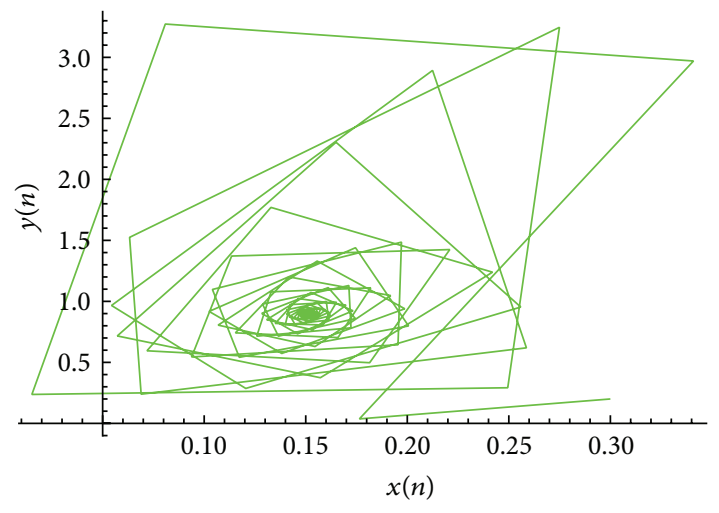

FIGURE 6: An attractor of the system (52).

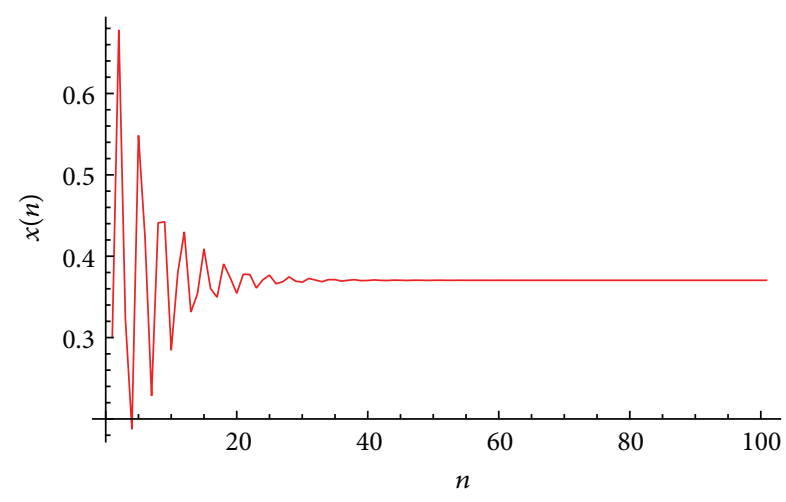

FIgURE 7: Plot of $x_{n}$ for the system (53).

global attractor is shown in Figure 9. The unique positive equilibrium point of the system (53) is given by $(\bar{x}, \bar{y})=$ $(0.370425,0.830832)$.

\section{Conclusion}

This work is related to the qualitative behavior of an exponential system of second-order rational difference equations. We have investigated the existence and uniqueness of positive steady-state of system (4). Under certain parametric conditions the boundedness and persistence of positive solutions are proved. Moreover, we have shown that unique positive equilibrium point of system (4) is locally as well as globally asymptotically stable. The main objective of dynamical systems theory is to predict the global behavior of a system based on the knowledge of its present state. An approach to this problem consists in determining the possible global behaviors of the system and determining which initial conditions lead to these long-term behaviors. Furthermore, rate of convergence of positive solutions of (4) which converge to its unique positive equilibrium point is demonstrated. Finally, some numerical examples are provided to support our theoretical results. These examples are experimental verification of our theoretical discussions.

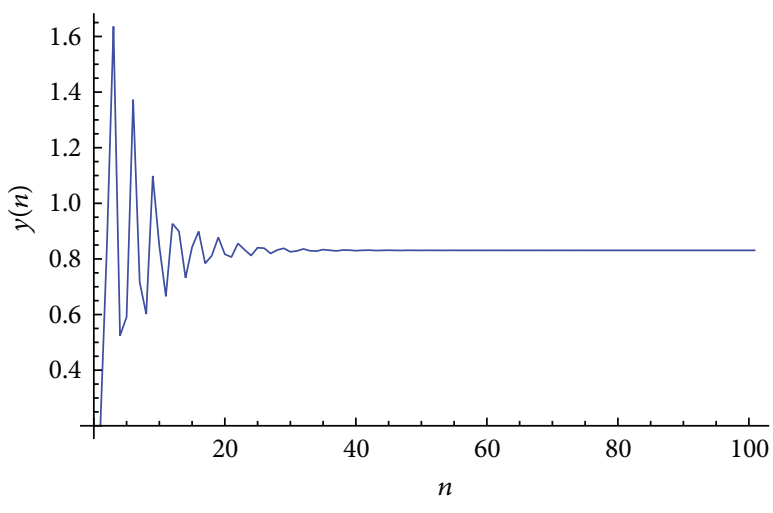

Figure 8: Plot of $y_{n}$ for the system (53).

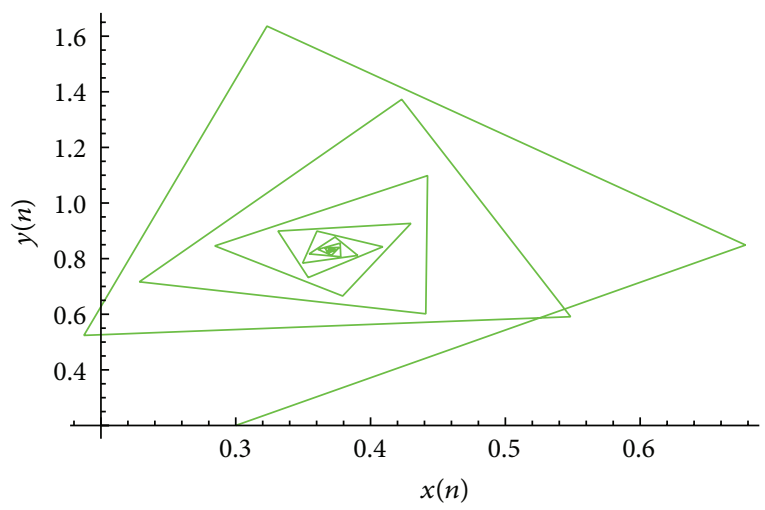

Figure 9: An attractor of the system (53).

\section{Conflict of Interests}

The authors declare that they have no conflict of interests regarding the publication of this paper.

\section{Acknowledgments}

The authors thank the main editor and anonymous referees for their valuable comments and suggestions leading to the improvement of this paper. This work was supported by the Higher Education Commission of Pakistan.

\section{References}

[1] Z. Zhou and X. Zou, "Stable periodic solutions in a discrete periodic logistic equation," Applied Mathematics Letters, vol. 16, no. 2, pp. 165-171, 2003.

[2] X. Liu, "A note on the existence of periodic solutions in discrete predator-prey models," Applied Mathematical Modelling. Simulation and Computation for Engineering and Environmental Systems, vol. 34, no. 9, pp. 2477-2483, 2010.

[3] E. El-Metwally, E. A. Grove, G. Ladas, R. Levins, and M. Radin, "On the difference equation $x_{n+1}=\alpha+\beta x_{n-1} e^{-x_{n}}$," Nonlinear Analysis: Theory, Methods \& Applications, vol. 47, pp. 46234634, 2001. 
[4] G. Papaschinopoulos, M. A. Radin, and C. J. Schinas, "On the system of two difference equations of exponential form: $x_{n+1}=$ $a+b x_{n-1} e^{-y_{n}}, y_{n+1}=c+d y_{n-1} e^{-x_{n}}$, " Mathematical and Computer Modelling, vol. 54, pp. 2969-2977, 2011.

[5] G. Papaschinopoulos and C. J. Schinas, "On the dynamics of two exponential type systems of difference equations," Computers \& Mathematics with Applications, vol. 64, no. 7, pp. 2326-2334, 2012.

[6] G. Papaschinopoulos, M. Radin, and C. J. Schinas, "Study of the asymptotic behavior of the solutions of three systems of difference equations of exponential form," Applied Mathematics and Computation, vol. 218, no. 9, pp. 5310-5318, 2012.

[7] I. Ozturk, F. Bozkurt, and S. Ozen, "On the difference equation $y_{n+1}=\left(\alpha+\beta e^{-y_{n}}\right) /\left(\gamma+y_{n-1}\right)$, , Applied Mathematics and Computation, vol. 181, no. 2, pp. 1387-1393, 2006.

[8] F. Bozkurt, "Stability analysis of a nonlinear difference equation," International Journal of Modern Nonlinear Theory and Application, vol. 2, pp. 1-6, 2013.

[9] H. Sedaghat, Nonlinear Difference Equations: Theory with Applications to Social Science Models, vol. 15, Kluwer Academic Publishers, Dordrecht, The Netherlands, 2003.

[10] V. L. Kocic and G. Ladas, Global Behavior of Nonlinear Difference Equations of Higher Order with Applications, Kluwer Academic Publishers, Dordrecht, The Netherlands, 1993.

[11] E. A. Grove and G. Ladas, Periodicities in Nonlinear Difference Equations, Chapman \& Hall/CRC, Boca Raton, Fla, USA, 2004.

[12] M. Pituk, "More on Poincare's and PERron's theorems for difference equations," Journal of Difference Equations and Applications, vol. 8, no. 3, pp. 201-216, 2002. 


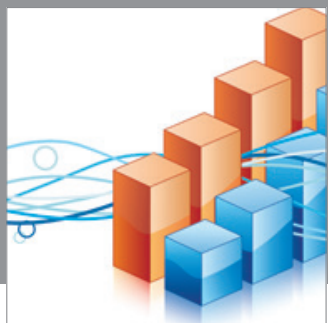

Advances in

Operations Research

mansans

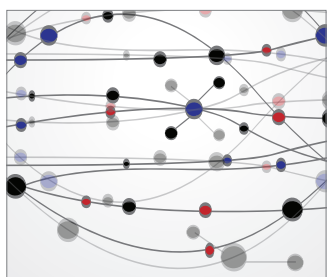

The Scientific World Journal
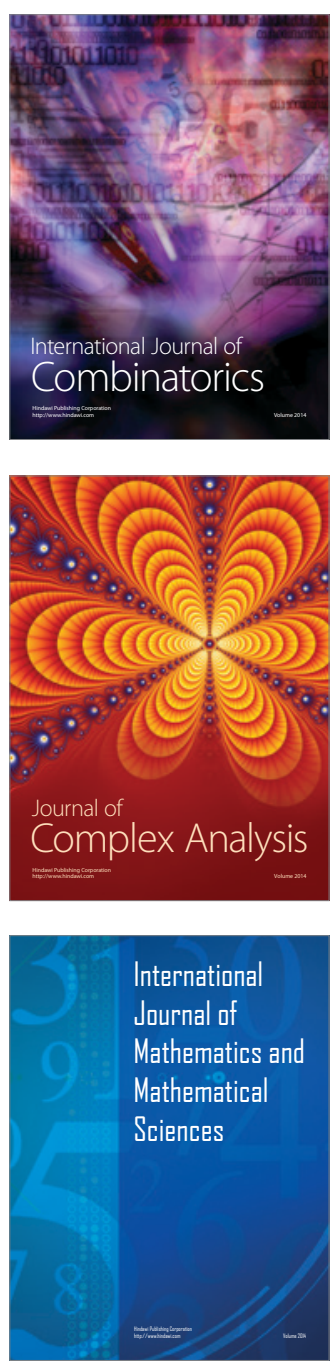
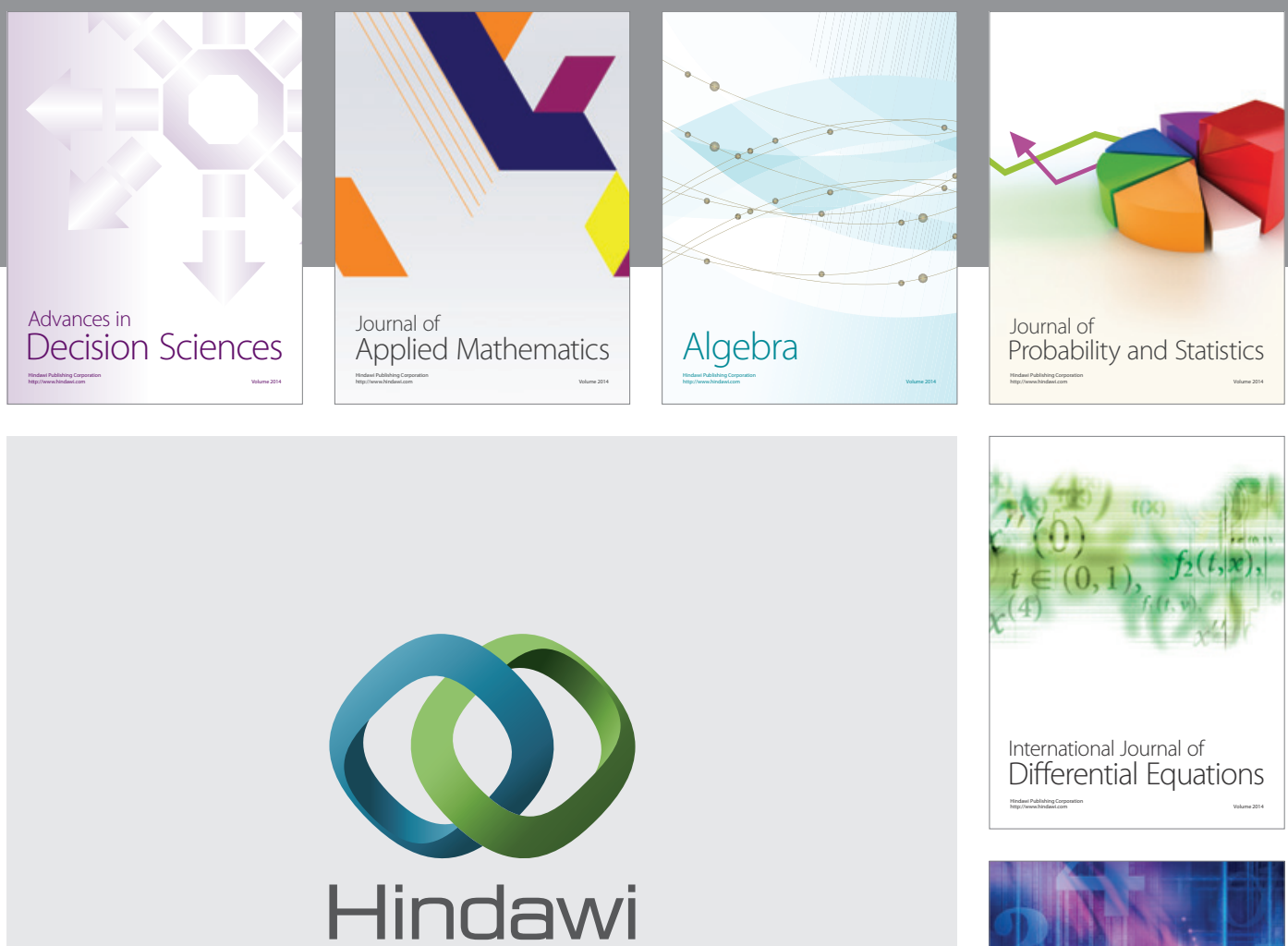

Submit your manuscripts at http://www.hindawi.com
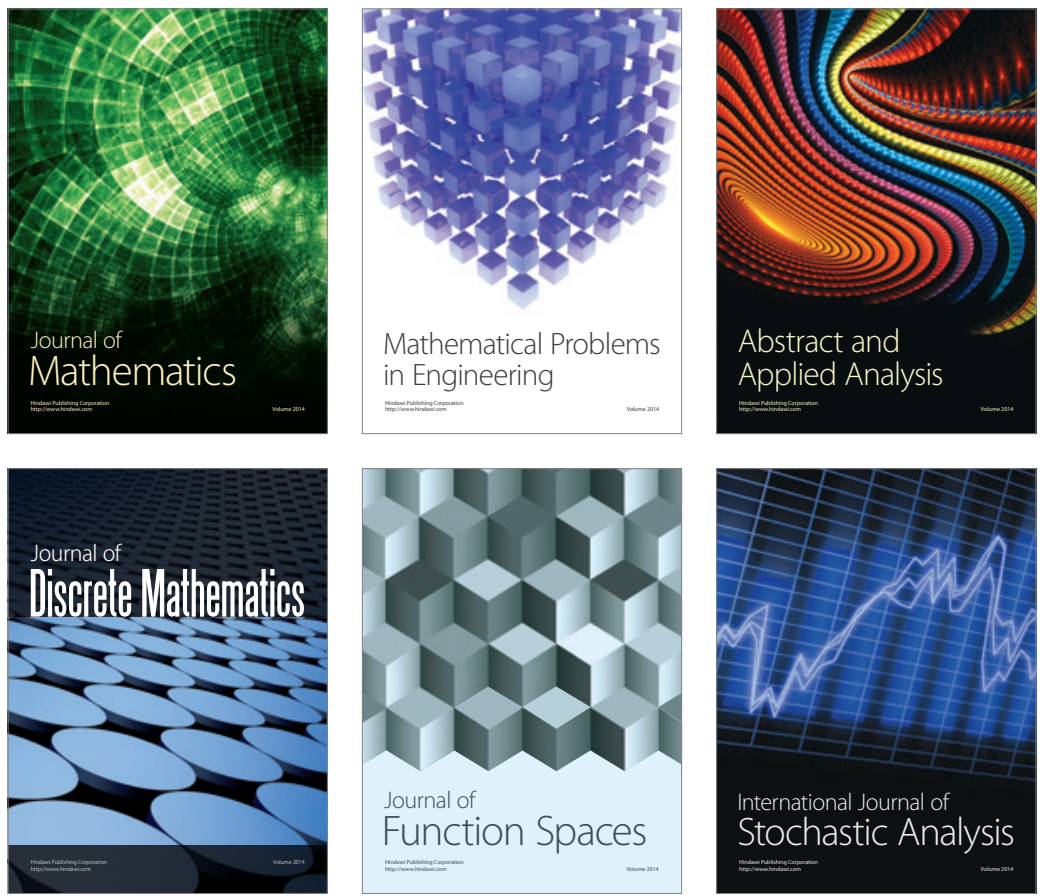

Journal of

Function Spaces

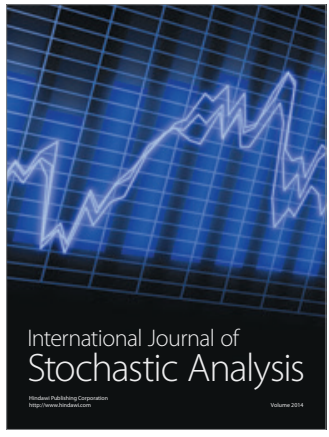

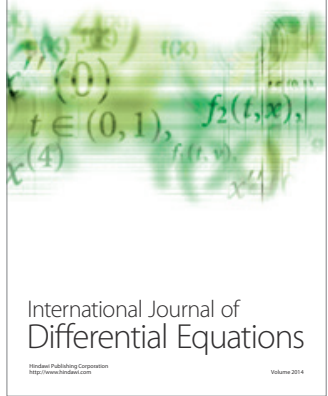
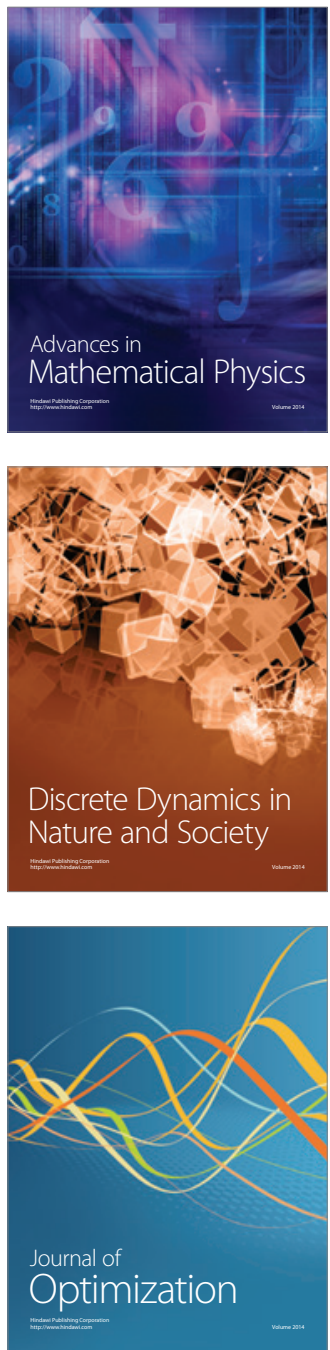